\title{
Regrowth of Carbon Nanotubes Array on Al Layer Coated Substrate
}

\author{
Chien-Chao Chiu, ${ }^{1}$ Masamichi Yoshimura, ${ }^{1}$ Kazuyuki Ueda, ${ }^{1}$ Yuya Kamizono, ${ }^{2}$ \\ Hisanori Shinohara, ${ }^{2}$ Yutaka Ohira, ${ }^{3}$ and Takayoshi Tanji ${ }^{3}$ \\ ${ }^{1}$ Graduate School of Engineering, Toyota Technological Institute, 2-12-1 Hisakata, Tempaku, Nagoya 468-8511, Japan \\ ${ }^{2}$ Department of Chemistry, Institute for Advanced Research, Nagoya University, Furo-cho, Chikusa, Nagoya 464 8602, Japan \\ ${ }^{3}$ Graduate School of Engineering, Nagoya University, Furo-cho, Chikusa, Nagoya 464 8603, Japan
}

Correspondence should be addressed to Masamichi Yoshimura, yoshi@toyota-ti.ac.jp

Received 5 June 2009; Revised 9 November 2009; Accepted 8 January 2010

Academic Editor: Do Kim

Copyright (C) 2010 Chien-Chao Chiu et al. This is an open access article distributed under the Creative Commons Attribution License, which permits unrestricted use, distribution, and reproduction in any medium, provided the original work is properly cited.

Carbon nanotube (CNT) arrays have been synthesized by a repeated growth method using a custom-fabricated plasma-enhanced thermal chemical vapor deposition (PE-thermal CVD) apparatus. The initial catalyst is a layered structure prepared by depositing $10 \mathrm{~nm}$ of $\mathrm{Al}$ followed by $3 \mathrm{~nm}$ of Fe on an oxidized silicon substrate. Following CNT growth, the CNT arrays are removed using an ultrasonic cleaner, and another CNT array is grown on the remaining Fe-Al bimetalic nanoparticles without the addition of more catalyst. Annealing the catalytic substrate in air between growth cycles results in the removal of residual amorphous carbon along with the CNTs, and oxidation of the Fe-Al nanoparticles. The diameter of CNTs is reduced with repeated growth-annealing cycles, an effect of which is attributed to the diminishing size of the catalytically active nanoparticles with each cycle. After two growth cycles, SWNTs with the extraordinarily narrow diameter of $0.86 \mathrm{~nm}$ are synthesized. The $I_{D} / I_{G}$ ratio derived from the Raman spectrum of these of the SWNT arrays shows the remarkably low value of 0.22 .

\section{Introduction}

Multi-walled carbon nanotubes (MWNTs) array [1, 2] has attracted increased attention because of its potential applications in gas sensor [3, 4], field emitter $[5,6]$ and ballistic devices [7-9]. The peculiar properties of singlewalled carbon nanotubes (SWNTs) [10-12] have also been regarded as one of the high-potential material for nanodevices. Thus, the SWNT array has recently gathered much attention. Control of size and density of catalytic particles is one of important factors for the fabrication of the SWNT array by chemical vapor deposition (CVD).

It is generally accepted that CNTs grow as carbon precipitates from supersaturated catalytic metal nanoparticles. The growth mechanisms are classified into base growth or tip growth, depending on whether the metal and nano-particle remains anchored at the support or not [13]. In a previous work [14], we demonstrated that the two mechanisms could occur at the same time, and hence the original catalytic nano-particle separates into two parts during growth. After removing the CNT arrays grown, a part of catalytic nanoparticle was left on the substrate, which can be used for subsequent CNT growth without additional catalyst. This repeated production of CNT array is called regrowth process, and the diameter of CNT can be reduced in accordance with the reduced catalyst size. By thermal annealing method, the initial $I_{D} / I_{G}$ ratio of 1.24 was improved to 0.41 in the fourth growth.

A supported Al layer has been reported to be critical in ensuring a high yield of SWNTs [12]. The Al layer promotes formation of very small bimetallic catalyst particles, consisting of $\mathrm{Al}$ and catalytic metal, for catalyzing the growth of SWNT $[11,12]$. It has been known that during annealing in vacuum, metal catalyst is efficiently gotten away from the surface and trapped in a possibly liquid Al-Si layer to keep the activity for CNT growth. AlOx layer, which could be formed from $\mathrm{Al}$ layer by annealing in $\mathrm{O}_{2}$ atmosphere, indeed 


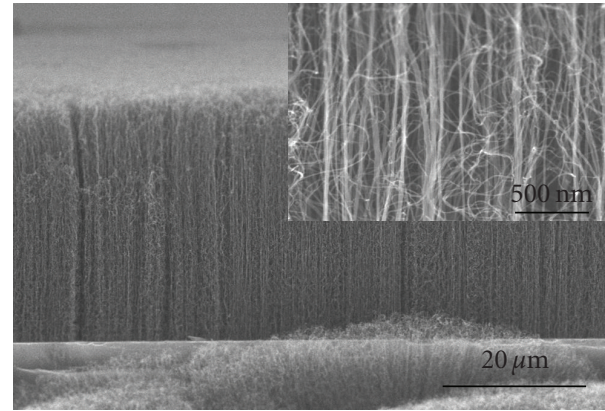

(a)

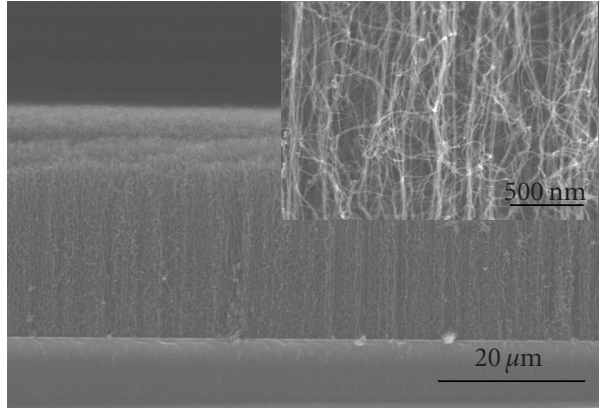

(b)

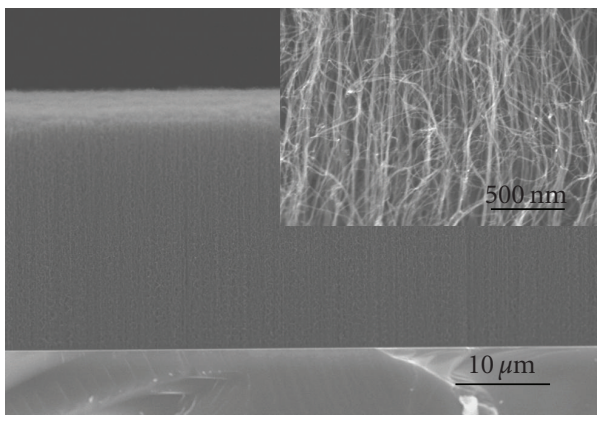

(c)

FIgURE 1: SEM images of CNT arrays grown after the (a) first, (b) second, and (c) third growth by the lift-off method. The length of CNT array is (a) 35, (b) 24 and (c) $25 \mu \mathrm{m}$. The insets show high-magnification SEM images.

promotes formation of thinner nanotubes as compared with other buffer layers [15].

In this paper, regrowth has been carried out by a custommade PE-thermal CVD, and CNTs formed at each regrowth cycle have been characterized. Two kinds of treatments, liftoff and annealing, are used to remove the CNT arrays. The variation of the diameter and length of CNT arrays for each cycle is characterized and compared. It is found that the thermal annealing is preferred to obtain uniform SWNT arrays with high purity.

\section{Experimental}

Carbon nanotubes were grown by a custom-made PEthermal CVD [16]. A $10 \mathrm{~nm}$ of Al film was coated on a $50 \mathrm{~nm}-\mathrm{SiO}_{2} / \mathrm{Si}$ substrate by thermal evaporation. Then a $3 \mathrm{~nm}$ of Fe film was deposited as a catalyst by arc plasma method. Then, $80 \mathrm{sccm}$ of $\mathrm{H}_{2}$ was exposed to the substrate at $700^{\circ} \mathrm{C}$ for 1 minute. For the CNT growth the flow rate of $\mathrm{H}_{2}$ and $\mathrm{CH}_{4}$ were 80 and $20 \mathrm{sccm}$, respectively. The total pressure was kept at 1.7 torr and the growth temperature was $800^{\circ} \mathrm{C}$.

The regrowth was carried out using two different processes: lift-off method and thermal annealing method. In the former the CNT array was removed in acetone by an ultrasonic cleaner. In the latter the array was set in the heating furnace in atmosphere at $650^{\circ} \mathrm{C}$ for 1 hour. This oxidation treatment removed amorphous carbon on the catalyst as well as CNTs. The surface morphology was observed by field emission scanning electron microscopy (FESEM, Hitachi, S-4700) and the crystallinity of CNTs was examined using $633 \mathrm{~nm}$ Raman spectroscopy.

Through the thermal annealing process the substrate was heated to $650^{\circ} \mathrm{C}$ in a CVD chamber, and the $\mathrm{Al}$ layer melted into small droplets. Residual oxygen inside the chamber or the underlying $\mathrm{SiO}_{2}$ layer reacts with $\mathrm{Al}$ and stable $\mathrm{AlO}_{\mathrm{X}}$ clusters are formed [17]. During the annealing at high temperature, the $\mathrm{AlO}_{\mathrm{X}}$ was transformed from $\mathrm{Al}$, which provides the support for the formation of Fe nanoparticles catalyzing the growth of SWNTs.

\section{Results and Discussion}

3.1. Lift-Off Process. Figures 1(a)-1(c) shows SEM images of CNT arrays grown by using lift-off treatment after the first, second and third growth, respectively. The lengths of CNT arrays are 35, 24, and $25 \mu \mathrm{m}$. The insets show magnified images. The diameters of CNTs, which are observed by transmission electron microscope (TEM) in Figures 2(a), 2 (b), and 2 (c), are $15.7 \pm 7.1,10.1 \pm 4.4$, and $7.9 \pm 3.6 \mathrm{~nm}$ for the first, second and third cycles, and were shown in, respectively. It seems that catalytic activity of nano-particles was preserved after the lift-off process. Figure 3(a) shows a SEM image of the substrate after removing CNT arrays by the lift-off method. The array was completely removed and donut-structure was observed on the surface. It seems that the hole inside the donut corresponds to the footprint of 


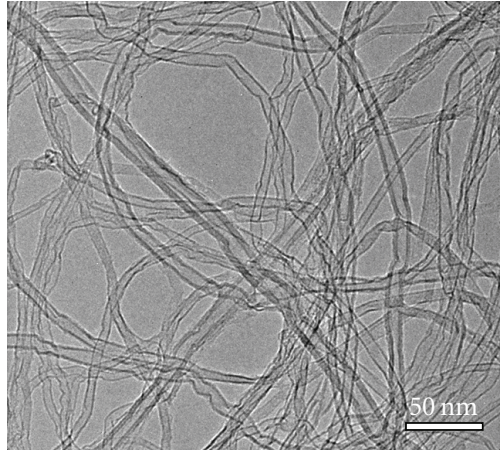

(a)

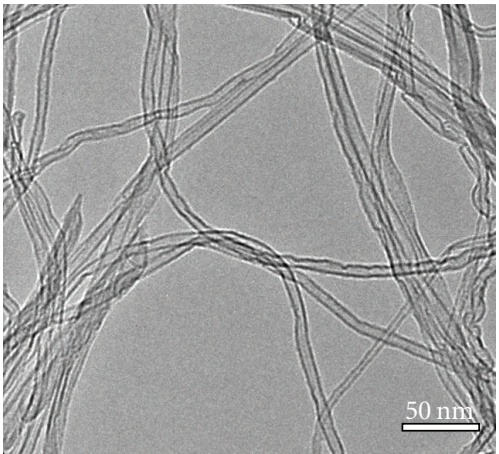

(d)



(b)

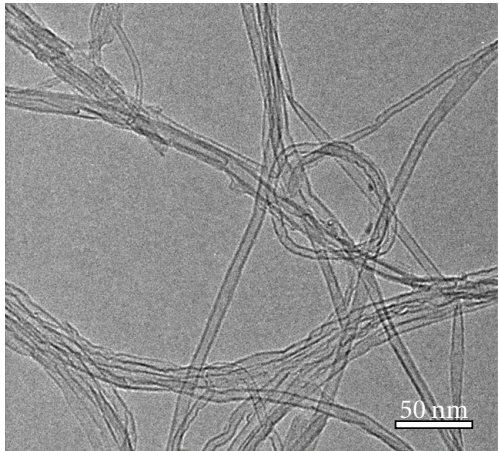

(e)

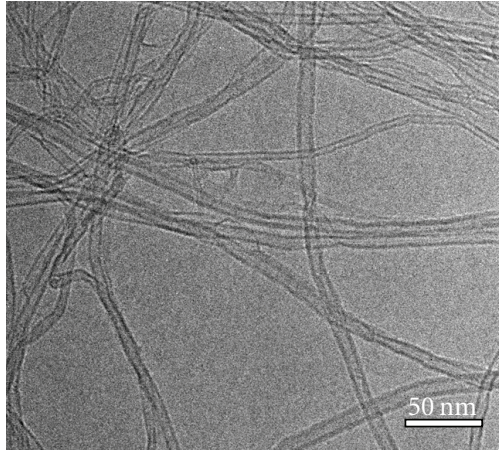

(c)



(f)

Figure 2: TEM images of CNT arrays grown after the (a) first, (b) second, and (c) third growth by the lift-off method, (d) second and (e) third growth by annealing method. (f) is the image of SWNTs bundle with the diameter of $0.86 \mathrm{~nm}$ after the third growth by annealing method.

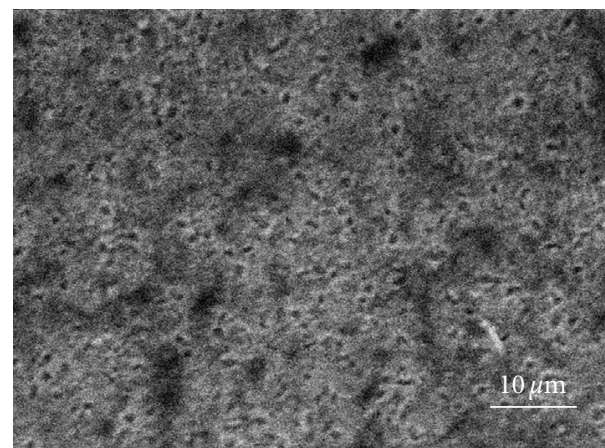

(a)



(b)

Figure 3: SEM images of the substrate surface after removing CNTs by (a) lift-off and (b) annealing method.

CNTs being removed, and that the donut to the residual amorphous carbon which was deposited during growth [14, 18].

Figure 4 shows Raman spectra of the CNT array obtained after each growth cycle by using the lift-off treatment. Similar $I_{D} / I_{G}$ ratios of 0.5 are observed, indicating that similar quality of CNTs was grown. This result also demonstrates that lift-off process is an admirable method for mass production of CNT arrays with similar length in any cycle of regrowth process.
3.2. Thermal Annealing Process. Figure 5 shows SEM images of CNT array grown after the first, second and third growth. The lengths of CNT arrays are 35, 22 and $24 \mu \mathrm{m}$, respectively. The TEM images present the average diameters of CNTs are $15.7 \pm 7.1,7.8 \pm 2.8$, and $6.3 \pm 2.4 \mathrm{~nm}$ for the first, second and third cycles, as shown in Figures 2(a), 2(d) and 2(e), respectively. Figure 3(b) shows the SEM image of the substrate after removal of CNT arrays by annealing. Uniform catalyst particles are isolated to each other on the substrate surface. From the result of successful regrowth, the catalytic 


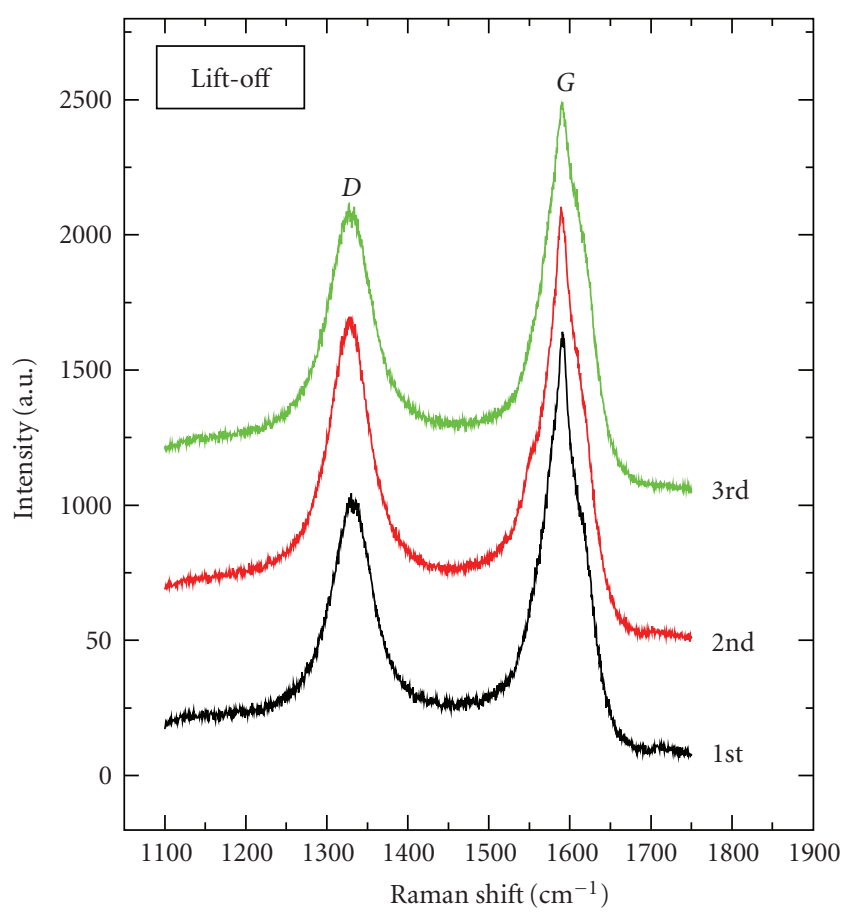

Figure 4: Raman spectra of CNTs at each cycle by the lift-off method.

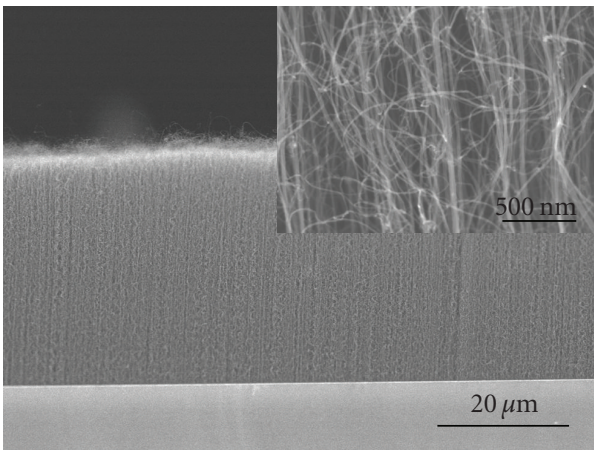

(a)

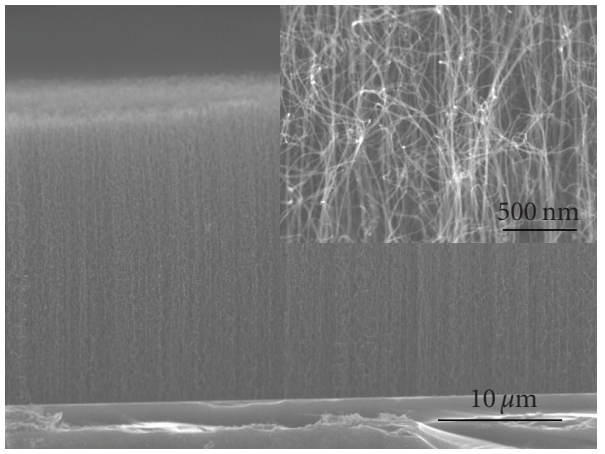

(b)



(c)

FIGURE 5: SEM images of CNT array grown for the (a) first, (b) second and (c) third growth by annealing method. The length of CNT array is (a) 35, (b) 22 and (c) $24 \mu \mathrm{m}$. The insets are high-magnification images. 


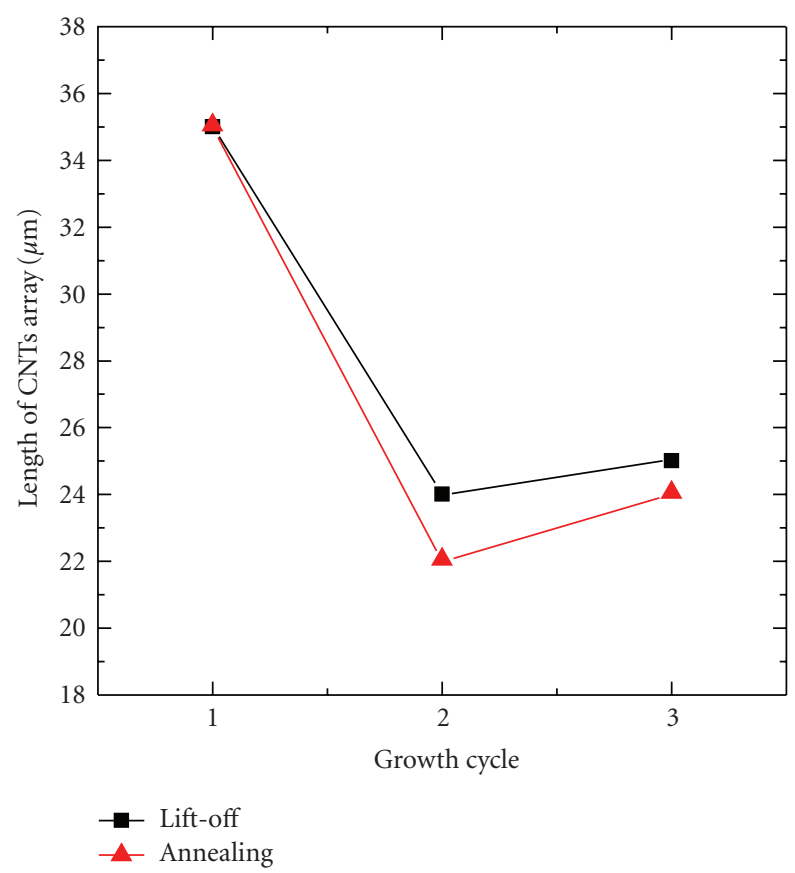

(a)

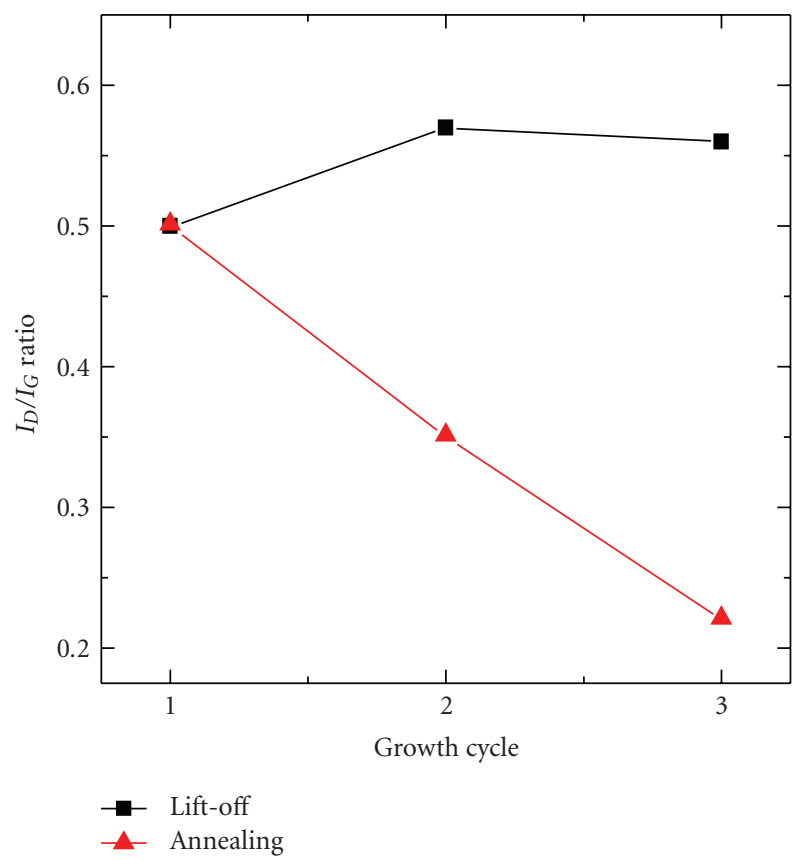

(b)

FIgURe 6: (a) CNT array length, and (b) $I_{D} / I_{G}$ ratio as a function of growth cycle.

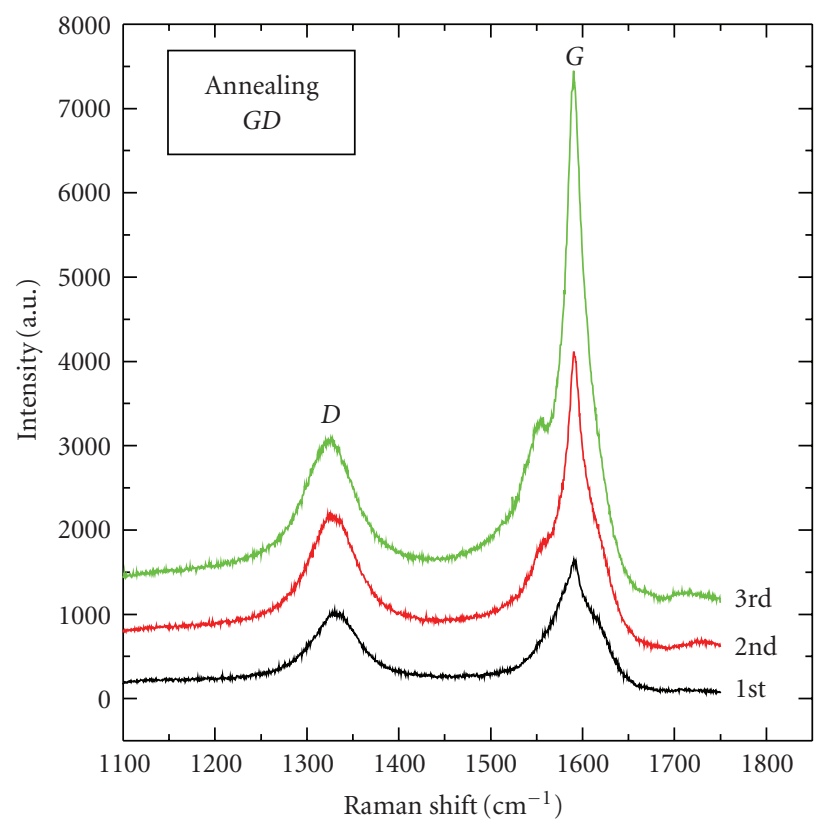

(a)

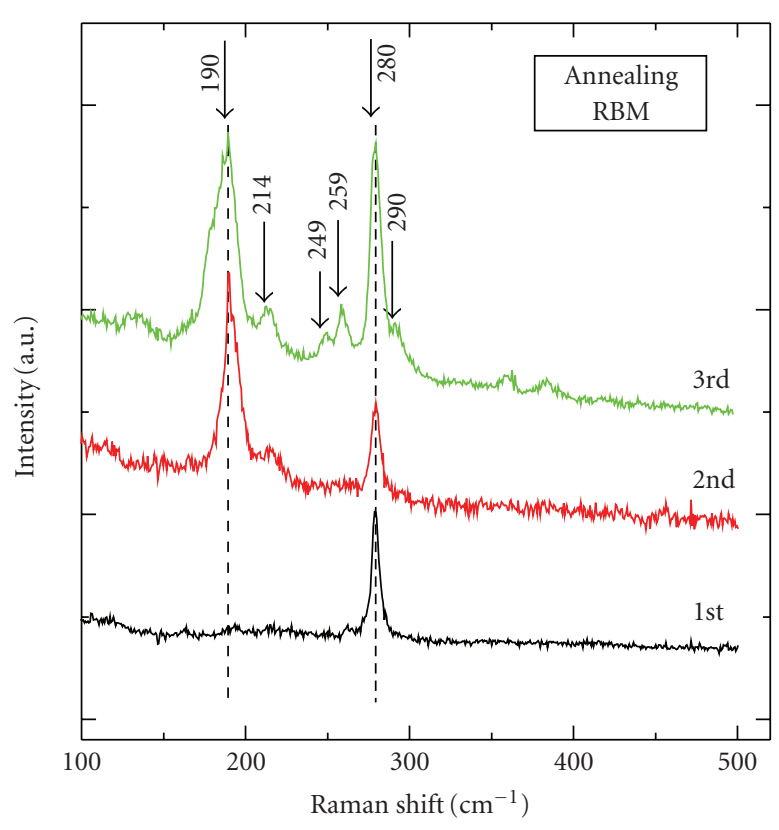

(b)

FIgURE 7: Raman spectra in (a) G-band and D-band, (b) RBM region of CNTs for three cycles growth by annealing method.

activity of nano-particles seems still alive for CNT regrowth and diameters are reduced after each regrowth cycle. The changes in the length of CNT arrays and growth cycle by the two treatments are shown in Figure 6(a).

Figure 7 shows Raman spectra of CNTs after each cycle. In Figure 7(a), a sharper G-band peak with higher intensity was observed as the growth cycle increased. The third growth cycle reaches a highest graphitic crystallization with the $I_{D} / I_{G}$ ratio of 0.22 from 0.5 grown by the first growth. The relationship between $I_{D} / I_{G}$ ratio and the growth cycles is shown in Figure 6(b). By using the lift-off treatment, the $I_{D} / I_{G}$ ratios after each cycle remain same $(0.5-0.57)$, indicative of uniform CNT arrays in every stage of growth. On the other hand, the $I_{D} / I_{G}$ ratio through thermal annealing 
decreases with growth cycle. It indicates the crystallization of CNTs was improved by thermal annealing and regrowth process. The key reason for the improvement of crystallization lies in the reduced diameter of CNT by the particle-separated regrowth process, which correlates with the quantity of defect structures in CNT. Although the Al layer could be partially oxidized through the lift-off process, Fe nanoparticle has still high possibility to contact with metallic Al. It is very hard to synthesize SWNTs on catalyst-Al bimetals [19]. Residual amorphous carbon was removed through annealing process. In addition, $\mathrm{Fe}$ and uncompletedly oxidized $\mathrm{Al}$ clusters were oxidized to $\mathrm{Fe}$ oxide and $\mathrm{AlO}_{\mathrm{X}}$. The catalyst particle is probably in contact with $\mathrm{AlO}_{\mathrm{X}}$ rather than with metallic Al cluster. Figure 7(b) presents a RBM region of the Raman spectrum. For the first growth, there is no SWNT peak shown in the spectrum. The only peak around $280 \mathrm{~cm}^{-1}$, representing the formation of iron oxide [20], was observed in this spectrum. In the spectrum of the second growth, another sharp peak around $190 \mathrm{~cm}^{-1}$ was observed. This indicates that SWNTs were produced by regrowth process and that the diameter was calculated to $1.31 \mathrm{~nm}$ [21]. It should be noted that not only MWNT array but SWNT array was synthesized by the regrowth using thermal annealing method. Five peaks 190, 214, 249, 259 and $290 \mathrm{~cm}^{-1}$ are found in the third growth spectrum, corresponding to the diameter of 1.31 , $1.16,1.00,0.96$ and $0.86 \mathrm{~nm}$, respectively. These values are smaller than $1.31 \mathrm{~nm}$ in the second growth. It indicates that the size of nanoparticles become smaller by each thermal annealing process on $\mathrm{AlO}_{\mathrm{X}}$ support layer. It should be also noted that the extraordinary narrow diameter of $0.86 \mathrm{~nm}$ was synthesized after the third growth, as clearly shown in the TEM image of Figure 2(f).

\section{Conclusions}

The CNT array was successfully grown by using a regrowth process without coating additional catalyst. By using liftoff treatment, the CNT array with uniform structure and length was regrown on the $\mathrm{Al}$ coated substrate. By using annealing treatment, SWNTs were found after the second growth due to reduction of catalytic particles on the $\mathrm{AlO}_{\mathrm{X}}$ support layer. After the third growth, extraordinary narrow diameter of $0.86 \mathrm{~nm}$ was synthesized through the third regrowth. Furthermore, the quality of CNTs was improved and extremely low $I_{D} / I_{G}$ ratio of 0.22 was obtained.

\section{Acknowledgment}

This work is supported by Strategic Research Foundation Grant-aided Project for private Universities: matching fund subsidy from MEXT (Ministy of Education, Culture, Sports, Science and Technology).

\section{References}

[1] X. Li, A. Cao, Y. J. Jung, R. Vajtai, and P. M. Ajayan, "Bottomup growth of carbon nanotube multilayers: unprecedented growth," Nano Letters, vol. 5, no. 10, pp. 1997-2000, 2005.
[2] C.-C. Chiu, T.-Y. Tsai, N.-H. Tai, and C.-Y. Lee, "Synthesis of ultra long vertically aligned carbon nanotubes using the rapid heating and cooling system in the thermal chemical vapor deposition process," Surface and Coatings Technology, vol. 200, no. 10, pp. 3215-3219, 2006.

[3] S. Sotiropoulou and N. A. Chaniotakis, "Carbon nanotube array-based biosensor," Analytical and Bioanalytical Chemistry, vol. 375, no. 1, pp. 103-105, 2003.

[4] K. Qian, T. Chen, B. Yan, et al., "Research on carbon nanotube array field emission pressure sensors," Electronics Letters, vol. 41, no. 14, pp. 824-825, 2005.

[5] C.-C. Chiu, T.-Y. Tsai, and N.-H. Tai, "Field emission properties of carbon nanotube arrays through the pattern transfer process," Nanotechnology, vol. 17, no. 12, pp. 2840-2844, 2006.

[6] A. Modi, N. Koratkar, E. Lass, B. Wei, and P. M. Ajayan, "Miniaturized gas ionization sensors using carbon nanotubes," Nature, vol. 424, no. 6945, pp. 171-174, 2003.

[7] A. Javey, J. Guo, D. B. Farmer, et al., "Carbon nanotube fieldeffect transistors with integrated ohmic contacts and high- $\kappa$ gate dielectrics," Nano Letters, vol. 4, no. 3, pp. 447-450, 2004.

[8] P. Avouris, "Molecular electronics with carbon nanotubes," Accounts of Chemical Research, vol. 35, no. 12, pp. 1026-1034, 2002.

[9] D. Dietzel, M. Faucher, A. Iaia, et al., "Analysis of mechanical properties of single wall carbon nanotubes fixed at a tip apex by atomic force microscopy," Nanotechnology, vol. 16, no. 3, pp. S73-S78, 2005.

[10] S. H. Tseng, N. H. Tai, W. K. Hsu, et al., "Ignition of carbon nanotubes using a photoflash," Carbon, vol. 45, no. 5, pp. 958964, 2007.

[11] R. Seidel, G. S. Duesberg, E. Unger, A. P. Graham, M. Liebau, and F. Kreupl, "Chemical vapor deposition growth of singlewalled carbon nanotubes at $600^{\circ} \mathrm{C}$ and a simple growth model," Journal of Physical Chemistry B, vol. 108, no. 6, pp. 1888-1893, 2004.

[12] C.-C. Chiu, C.-Y. Chen, N.-H. Tai, and C.-H. Tsai, "Growth of high-quality single-walled carbon nanotubes through the thermal chemical vapor deposition using co-sputtering Fe-Mo films as catalysts," Surface and Coatings Technology, vol. 200, no. 10, pp. 3199-3202, 2006.

[13] V. Vinciguerra, F. Buonocore, G. Panzera, and L. Occhipinti, "Growth mechanisms in chemical vapour deposited carbon nanotubes," Nanotechnology, vol. 14, no. 6, pp. 655-660, 2003.

[14] C.-C. Chiu, M. Yoshimura, and K. Ueda, "Regrowth of carbon nanotube array by microwave plasma-enhanced thermal chemical vapor deposition," Japanese Journal of Applied Physics, vol. 47, no. 4, part 1, pp. 1952-1955, 2008.

[15] T. de los Arcos, Z. M. Wu, and P. Oelhafen, "Is aluminum a suitable buffer layer for carbon nanotube growth?" Chemical Physics Letters, vol. 380, no. 3-4, pp. 419-423, 2003.

[16] C.-C. Chiu, M. Yoshimura, and K. Ueda, "Synthesis of carbon nanotubes by microwave plasma-enhanced hot filament chemical vapor deposition," Diamond and Related Materials, vol. 17, no. 4-5, pp. 611-614, 2008.

[17] R. Y. Zhang, I. Amlani, J. Baker, J. Tresek, and R. K. Tsui, "Chemical vapor deposition of single-walled carbon nanotubes using ultrathin Ni/Al film as catalyst," Nano Letters, vol. 3, no. 6, pp. 731-735, 2003.

[18] M. Hiramatsu, H. Nagao, M. Taniguchi, H. Amano, Y. Ando, and M. Hori, "High-rate growth of films of dense, aligned double-walled carbon nanotubes using microwave plasmaenhanced chemical vapor deposition," Japanese Journal of Applied Physics, vol. 44, no. 20-23, pp. L693-L695, 2005. 
[19] R. Seidel, M. Liebau, G. S. Duesberg, et al., "In-situ contacted single-walled carbon nanotubes and contact improvement by electroless deposition," Nano Letters, vol. 3, no. 7, pp. 965-968, 2003.

[20] M. A. Legodi and D. de Waal, "Raman spectroscopic study of ancient South African domestic clay pottery," Spectrochimica Acta Part A, vol. 66, no. 1, pp. 135-142, 2007.

[21] A. Jorio, R. Saito, J. H. Hafner, et al., "Structural $(n, m)$ determination of isolated single-wall carbon nanotubes by resonant Raman scattering," Physical Review Letters, vol. 86, no. 6, pp. 1118-1121, 2001. 

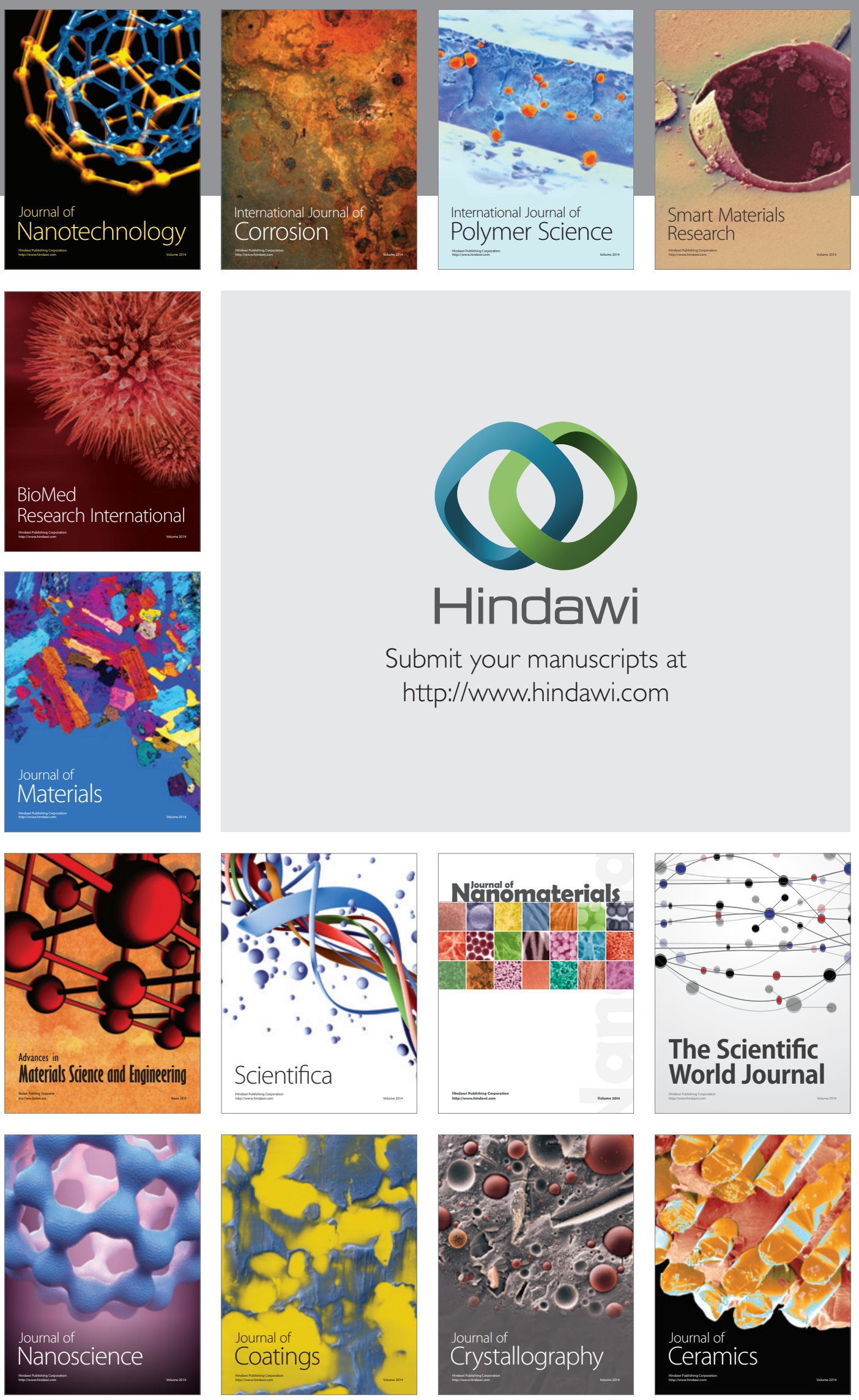

The Scientific World Journal

Submit your manuscripts at

http://www.hindawi.com

\section{World Journal}



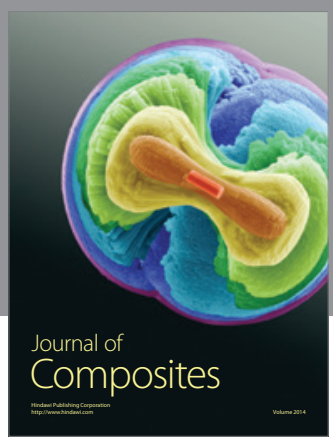
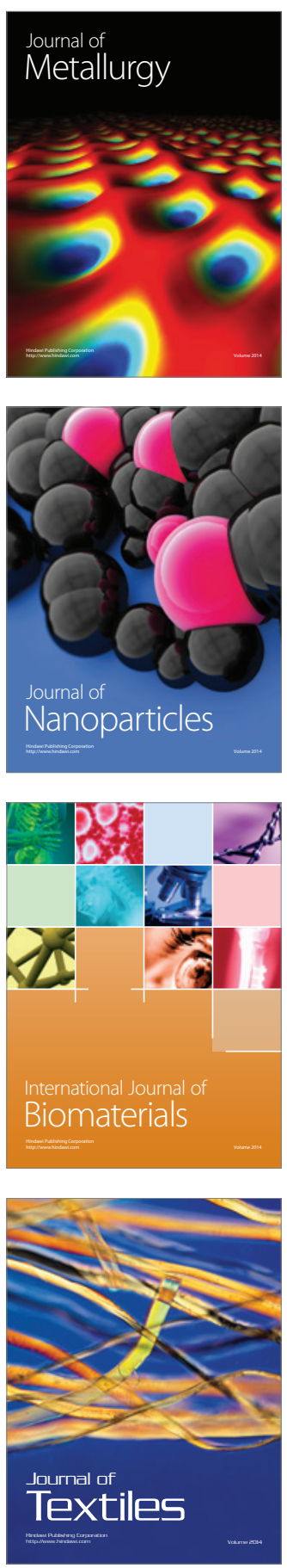